\title{
Improved Determination of Surface and Atmospheric Temperatures Using Only Shortwave AIRS Channels
}

\author{
Joel Susskind $^{1}$, John Blaisdell ${ }^{2}$, and Lena Iredell ${ }^{2}$ \\ ${ }^{1}$ NASA Goddard Space Flight Center, Greenbelt, MD, USA 20771 \\ ${ }^{2}$ SAIC, NASA Goddard Space Flight Center, Greenbelt, MD, USA 20771
}

\begin{abstract}
AIRS was launched on EOS Aqua on May 4, 2002, together with AMSU-A and HSB, to form a next generation polar orbiting infrared and microwave atmospheric sounding system. AIRS is a grating spectrometer with a number of linear arrays of detectors with each detector sensitive to outgoing radiation in a characteristic frequency $\boldsymbol{v}_{\boldsymbol{i}}$ with a spectral band pass $\Delta v_{i}$ of roughly $v_{i} / 1200$. AIRS contains 2378 spectral channels covering portions of the spectral region $650 \mathrm{~cm}^{-1}$ $(15.38 \mu \mathrm{m})-2665 \mathrm{~cm}^{-1}(3.752 \mu \mathrm{m})$. These spectral regions contain significant absorption features from two $\mathrm{CO}_{2}$ absorption bands, the $15 \mu \mathrm{m}$ (longwave) $\mathrm{CO}_{2}$ band, and the $4.3 \mu \mathrm{m}$ (shortwave) $\mathrm{CO}_{2}$ absorption band. There are also two atmospheric window regions, the $12 \mu \mathrm{m}-8 \mu \mathrm{m}$ (longwave) window, and the $4.17 \mu \mathrm{m}-3.75 \mu \mathrm{m}$ (shortwave) window. Historically, determination of surface and atmospheric temperatures from satellite observations was performed using primarily observations in the longwave window and $\mathrm{CO}_{2}$ absorption regions. One reason for this was concerns about the effects, during the day, of reflected sunlight and non-Local Thermodynamic Equilibrium (non-LTE) on the observed radiances in the shortwave portion of the spectrum. According to cloud clearing theory, more accurate soundings of both surface skin and atmospheric temperatures can be obtained under partial cloud cover conditions if one uses the longwave channels to determine cloud cleared radiances $\hat{R}_{i}$ for all channels, and uses $\hat{R}_{i}$ only from shortwave channels in the determination of surface and atmospheric temperatures. This procedure is now being used by the AIRS Science Team in preparation for the AIRS Version 6 Retrieval Algorithm. This paper describes how the effects on the radiances of solar radiation reflected by clouds and the Earth's surface, and also of non-LTE, are accounted for in the analysis of the data. Results are presented for both daytime and nighttime conditions showing improved surface and atmospheric soundings under partial cloud cover resulted from not using $\hat{R}_{i}$ in the retrieval process for any longwave channels sensitive to cloud effects. This improvement is made possible because AIRS NEDT in the shortwave portion of the spectrum is extremely low.
\end{abstract}

Keywords: AIRS/AMSU, high spectral resolution IR sounders, retrieval methodology, IR sounding in cloudy conditions, cloud cleared radiances, Quality Control.

\section{INTRODUCTION}

AIRS was launched on EOS Aqua on May 4, 2002, together with AMSU-A and HSB, to form a next generation polar orbiting infrared and microwave atmospheric sounding system ${ }^{1}$. AIRS is a grating spectrometer with a number of linear arrays of detectors with each detector sensitive to outgoing radiation in a characteristic frequency $v_{i}$ with a spectral band pass $\Delta v_{i}$ of roughly $v_{i} / 1200$. AIRS contains 2378 spectral channels covering portions of the spectral region $650 \mathrm{~cm}^{-1}$ $(15.38 \mu \mathrm{m})-2665 \mathrm{~cm}^{-1}(3.752 \mu \mathrm{m})$. The spectral sampling interval (except for the existence of a few gaps) is $v_{i} / 2400$, giving two AIRS channels per spectral half width. AIRS is accompanied by the microwave instrument AMSU-A. There is a $3 \times 3$ array of AIRS footprints within a given AMSU-A footprint, with spatial resolutions of $13 \mathrm{~km}$ and $45 \mathrm{~km}$ at nadir viewing respectively.

Figure 1 shows NEDT in blue for AIRS channels evaluated at the channel brightness temperatures $\Theta_{i}$ for a clear sky tropical scene. Figure 1 also includes the noise in red for the IASI instrument evaluated at IASI $\Theta_{i}$ for the same scene. IASI was launched on October 19, 2006 on MetOp-A, also accompanied by AMSU-A ${ }^{2}$. IASI has 4 footprints within an AMSU-A footprint. IASI is an interferometer with a maximum Optical Path Displacement (OPD) of $2.0 \mathrm{~cm}$. The spectral sampling of IASI is $0.25 \mathrm{~cm}^{-1}$ throughout the spectrum. This is roughly the same as AIRS at $650 \mathrm{~cm}^{-1}$, and about 4 times more often than AIRS at $2400 \mathrm{~cm}^{-1}$. Channel noise for AIRS and IASI is comparable throughout most of the spectrum but IASI channel noise is very large relative to AIRS in the shortwave portion of the spectrum. In principle, better noise 
performance could have been obtained by IASI at these short wavelengths. Channels in this portion of the spectrum were not properly utilized by other researchers in the retrieval process because of concerns about effects on the observed radiances, during the day, of solar radiation reflected by both the surface and clouds, as well as effects of non-Local Thermodynamic Equilibrium (non-LTE). Therefore, noise performance in this portion of the spectrum was not a priority in the design of IASI. The methodology we use in this paper to determine improved surface and atmospheric temperature using only shortwave AIRS observations is not practical using IASI because the IASI noise performance is not adequate in the shortwave portion of the spectrum.

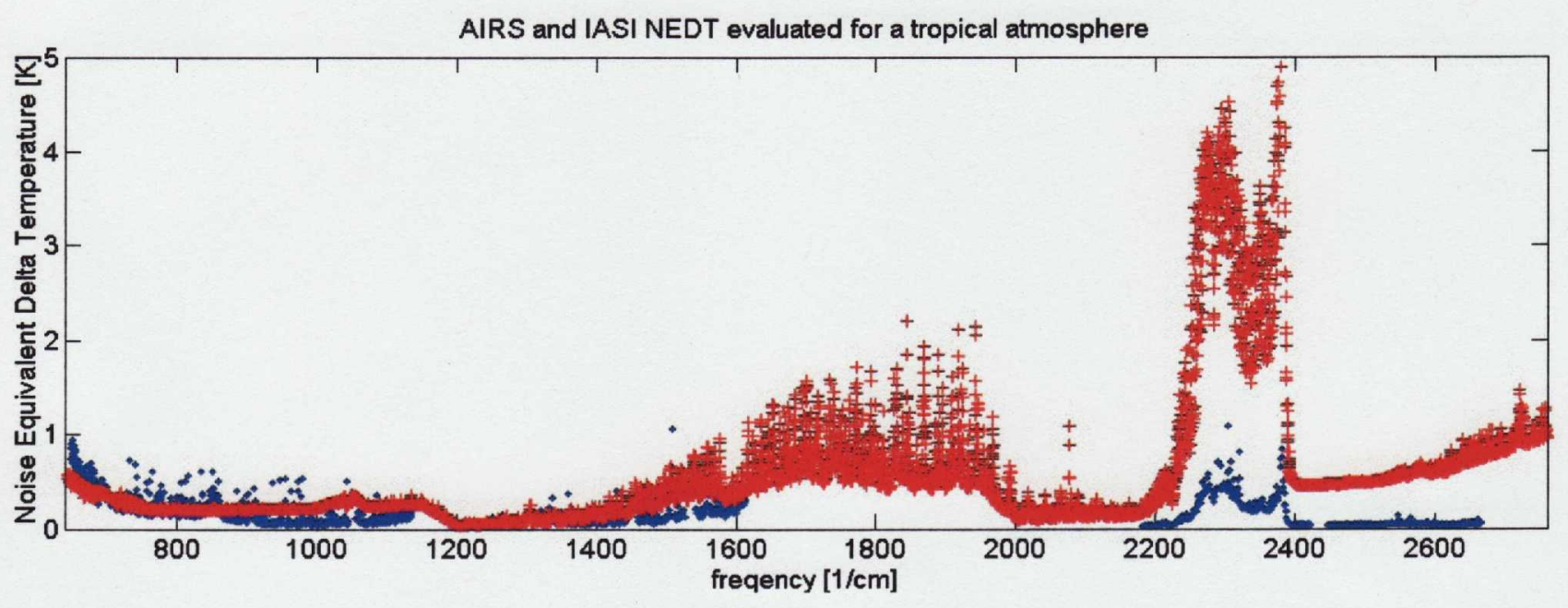

Figure 1

The sounding goals of AIRS are to produce $1 \mathrm{~km}$ tropospheric layer mean temperatures with an rms error of $1 \mathrm{~K}$, and 1 $\mathrm{km}$ layer precipitable water with an rms error of 20 percent, in cases with up to 80 percent effective cloud cover. The primary products of AIRS/AMSU-A are twice daily global fields of atmospheric temperature-humidity profiles, ozone profiles, sea/land surface skin temperature, and cloud related parameters including OLR. Also included are the reconstructed clear column radiances $\hat{R}_{i}$ used to generate these products. $\hat{R}_{i}$ is a derived quantity representative of the radiance AIRS would have seen in channel $i$ if there were no clouds in the field of view. All products also have error estimates. The products are designed for data assimilation purposes so as to improve numerical weather prediction, as well as for the study of climate and meteorological processes. With regard to data assimilation, one can use either the products themselves or the clear column radiances from which the products were derived. From both the data assimilation and climate perspectives, better spatial coverage of the retrievals is desirable provided the accuracy of retrieved products is sufficient.

The theoretical approach used to analyze AIRS/AMSU/HSB data in the presence of clouds in the AIRS Science Team Version 3 at-launch algorithm, and that used in the Version 4 post-launch algorithm, have been published previously ${ }^{3,4}$. Significant theoretical and practical improvements have been made in the analysis of AIRS/AMSU data since the Version 4 algorithm. Most of these have already been incorporated in the AIRS Science Team Version 5 algorithm ${ }^{5}$, now being used operationally at the Goddard DISC. The AIRS Version 5 retrieval algorithm contained three significant improvements over Version 4. Improved physics in Version 5 allowed for use of AIRS clear column radiances $\hat{R}_{i}$ in the entire $4.3 \mu \mathrm{m} \mathrm{CO}_{2}$ absorption band in the retrieval of temperature profiles $\mathrm{T}(\mathrm{p})$ during both day and night. Tropospheric sounding $15 \mu \mathrm{m} \mathrm{CO}$ observations were used primarily in the generation of clear column radiances $\hat{R}_{i}$ for all channels. This new approach allowed for the generation of accurate Quality Controlled values of $\hat{R}_{i}$ and $T(p)$ under more stressing cloud conditions. Secondly, Version 5 contained a new methodology to provide accurate case-by-case error estimates for retrieved geophysical parameters and for channel-by-channel clear column radiances. Thresholds of these error estimates were used in the new approach for Quality Control. Finally, Version 5 contained for the first time an approach to provide AIRS soundings in partially cloudy conditions that does not require use of any microwave data. This new AIRS Only sounding methodology, referred to as AIRS Version $5 \mathrm{AO}$, was developed as a backup to AIRS Version 5 should the 
AMSU-A instrument fail. Susskind et al. ${ }^{5}$ show that Version 5 AIRS Only soundings are only slightly degraded from the AIRS/AMSU soundings, even at large fractional cloud cover.

Significant further progress has been made by the AIRS Science Team since the delivery of the AIRS Version 5 retrieval algorithm in preparation of the AIRS Science Team Version 6 AIRS/AMSU retrieval algorithm expected to be made operational at the Goddard DISC in early 2010. The current version of the retrieval algorithm, as of July 2009, is referred to in this paper as Version 5.24. Version 5.24 is very similar to Version 5 with one major exception. As in Version 5, the generation of cloud cleared radiances $\hat{R}_{i}$ for all channels is performed using observed radiances $R_{i}$ for longwave $15 \mu \mathrm{m}$ and $11 \mu \mathrm{m}$ channels. Tropospheric temperature profiles were retrieved in Version 5 using only the AIRS shortwave 4.2 $\mu \mathrm{m} \mathrm{CO} 2$ channels, but surface skin temperature was retrieved simultaneously with surface spectral emissivity and bidirectional reflectance using observations both in the longwave $8-12 \mu \mathrm{m}$ window region and in the shortwave $4.0 \mu \mathrm{m}-$ $3.76 \mu \mathrm{m}$ window region. In Version 5.24, only window observations in the shortwave window region, $4.0 \mu \mathrm{m}-3.76 \mu \mathrm{m}$ are used to determine surface skin temperatures as well as shortwave surface spectral emissivities and surface bidirectional reflectance. The current use of only shortwave AIRS channels in the retrieval of both atmospheric and surface parameters has resulted in significant improvement in the ability to obtain both accurate temperature profiles and surface skin temperatures under more stressing partial cloud cover conditions. This paper discusses some of the theoretical improvements made both in Version 5 as compared to Version 4, and Version 5.24 compared to Version 5.

\section{OVERVIEW OF THE AIRS RETRIEVAL METHODOLOGY}

The AIRS Science Team Version 5, Version 5 AO, and Version 5.24 retrieval algorithms are very similar to each other and to Versions of the AIRS Science Team retrieval algorithms described previously ${ }^{3,4}$. The basic approach and methodology used to determine geophysical parameters from observed AIRS/AMSU radiances is identical to that described in great detail in ${ }^{3}$. The basic approached is summarized below.

Retrievals of all geophysical parameters are physically based and represent states $X_{j}$ determined for case c that best match a set of clear column radiance $\hat{R}_{i, c}$ for the subset of AIRS channels $i$ used in the retrieval process. The clear column radiances $\hat{R}_{i, c}$ are a derived quantity that represent the radiances that AIRS "would have seen" if the $3 \times 3$ AIRS field of regard $(\mathrm{FOR})_{\mathrm{c}}$ were completely clear. Retrievals of geophysical parameters are performed sequentially, that is, only a subset of the geophysical parameters within the state $X_{j}$ is modified from that of the incoming state $X_{j}^{0}$ in a given step. A GCM forecast is not used in any way in the retrieval procedure, except for the forecasted surface pressure $p_{\text {surf }}$, which is used as the lower pressure boundary when computing expected radiances $R_{i, C L R}$ for a given geophysical state.

In Version 5, the steps are as follows: After a start up procedure giving the state used to determine the initial clear column radiances $\hat{R}_{i}^{0}$, AIRS/AMSU data are used to retrieve: a) surface skin temperature, surface spectral emissivity and surface bi-directional reflectance of solar radiation; b) atmospheric temperature profile; c) atmospheric moisture profile; d) atmospheric ozone profile; e) atmospheric $\mathrm{CO}$ profile; f) atmospheric $\mathrm{CH}_{4}$ profile; and g) cloud properties. These steps are done sequentially, solving only for the variables to be determined in each retrieval step and using previously determined variables as fixed but with an appropriate uncertainty attached to them which is accounted for in the channel noise covariance matrix $\mathrm{N}$. The objective in each step (a-f) is to find solutions which best match $\hat{R}_{i}$ for the subset of channels selected for use in that step, bearing in mind the channel noise estimates. Steps a-f are ordered so as to allow for selection of channels in each step which are primarily sensitive to variables to be determined in that step or determined in a previous step, and relatively insensitive to other parameters. Separation of the problem in this manner also allows for the problem in each step to be made as linear as possible. Step $g$ is performed after the surface and atmospheric conditions have been determined using the observed radiances $R_{i}$.

In Version 5.24, there is a slight modification to the sequence of steps used in Version 5, as there is a new step performed in the retrieval sequence. In Version 5, step a) used channels in both the longwave and shortwave window regions and simultaneously solved for surface skin temperature $T_{s}$, shortwave surface spectral emissivity $\varepsilon_{s w}(v)$ and surface spectral bi-directional reflectance $\rho_{s w}(v)$, and longwave surface spectral emissivity $\varepsilon_{\ell w}(v)$. In Version 5.24, only shortwave window channels are used in this retrieval step to simultaneously determine $T_{s}, \varepsilon_{s w}(v)$, and $\rho_{s w}(v)$. The 
longwave surface spectral emissivity $\varepsilon_{\ell w}(\nu)$ is now solved for in a subsequent step using only channels in the longwave window spectral region. This new step is performed after the humidity profile retrieval step because longwave window radiances can be very sensitive to the amount of atmospheric water vapor.

\section{BASIC THEORETICAL CONSIDERATIONS FOR CLOUD CLEARING}

Using the assumption that adjacent fields of view have otherwise identical geophysical conditions except for cloud cover, Chahine ${ }^{6}$ has shown that in the case of K-1 cloud formations, observations in K fields of view (FOV's) are needed to obtain channel $i$ cloud cleared radiances $\hat{R}_{i}$ which can be determined using the equation

$$
\hat{R}_{i}=R_{i, 1}+\sum_{k=1}^{K-1} \eta_{k}\left(R_{i, 1}-R_{i, K+1-k}\right)
$$

In which $R_{i, k}$ is the channel $i$ radiance observation in field of view $k$.

In analyzing AIRS/AMSU-A data, a single sounding is produced using all 9 AIRS FOV's falling within a single AMSUA footprint ${ }^{3,4}$. The AIRS Science Team has found it is advantageous to extrapolate the radiances in the 9 fields of view according to a similar equation of the form

$$
\hat{R}_{i}^{n}=R_{i, A V G}+\sum_{k=1}^{9} \eta_{k}^{n}\left(R_{i, A V G}-R_{i, k}\right)
$$

where $R_{i, A V G}$ is the average channel i radiance of all 9 fields of view. The superscript $n$ has been added to Equation 2 because the generation of $\hat{R}_{i}$ is iterative ${ }^{5}$. Optimal values of $\eta_{k}^{n}$ will give true values of $\hat{R}_{i}$ up to instrumental noise effects. While there are 9 values of $\eta_{k}$ shown in Equation 9 , only 8 of them are linearly independent.

Most of the $I=59$ cloud clearing channels used in Equation 2 are channels in the $15 \mu \mathrm{m} \mathrm{CO}$ absorption band with temperature weighting functions covering the atmosphere from the lower stratosphere to the surface. Observations in some longwave window channels are used as well as the determination of $\eta_{k}^{n}$.

\subsection{Determination of $\boldsymbol{\eta}$}

If, for each channel $i$, one substitutes an estimate of $R_{i, C L R}^{n}$ for $\hat{R}_{i}^{n}$ in Equation 2, this gives $I$ equations for $K(=9)$ unknowns. The unconstrained weighted least square solution to this multilinear problem is given by

$$
\eta_{K x 1}^{n}=\left[\Delta R^{\prime} N^{-1} \Delta R\right]_{K x K}^{-1} \Delta R^{\prime} N^{-1} \Delta R_{C L R}^{n}
$$

where $\Delta R$ is an IxK matrix with $\Delta R_{i, k}=R_{A V G}-R_{i, k}, \Delta R_{C L R}^{n}$ is an Ix1 matrix given by $\Delta R_{i, C L R}^{n}=R_{i, C L R}^{n}-R_{i, A V G}$, and $N$ is an IxI channel noise covariance matrix. $R_{i, C L R}^{n}$ is generated by computing expected radiances for cloud clearing channel ${ }_{\mathrm{i}}$ based on the current estimate of the geophysical state $X^{n}$ assuming cloud free conditions. As in Susskind et al. $^{3,4}$, the solution for $\eta$ is stabilized by solving for coefficients of up to the first four principal components of $\left[\Delta R^{\prime} N^{-1} \Delta R\right]$. The parameters $\eta_{k}$ determined in Equation 3, using I channels, are assumed to characterize the cloud formations and thus should be valid for use in all channels.

The key to the accurate determination of $\eta$ is obtaining the best estimates of $\Delta R_{i, C L R}^{n}$, along with an accurate treatment of the noise covariance matrix $\mathrm{N}$. The values of $\Delta R_{i, C L R}^{n}$, which are used to determine $\eta$ (and $\hat{R}_{i}$ ) are iterative and are computed based on the current best estimate of all relevant surface and atmospheric properties. It is best for the estimated geophysical parameters to be unbiased over large regions of the atmosphere. In all previous versions of the cloud clearing algorithm, including Version 4 , the geophysical state $X^{0}$ used to estimate $R_{i, C L R}^{0}$ was derived from an AMSU-A retrieval state, thus insuring an unbiased temperature and moisture profile over coarse layers in the atmosphere. Subsequent research has shown that a reasonably good regression relationship can be obtained between geophysical parameters and observed (un-cloud cleared) AIRS radiances, and this regression based state can be used to generate an 
alternative initial state $X^{0}$ used for initial cloud clearing. Generation of this state $X^{0}$ does not require use of any AMSU observations. This is the approach used to obtain $X^{0}$ in the AIRS Only cloud clearing system, Version $5 \mathrm{AO}$. Version 5 uses analogous methodology to give the initial cloud clearing state, but the cloudy regression makes use of both AIRS and AMSU observations. The state derived from this cloudy regression, $X^{\text {reg }}$ is followed in Version 5 by an AMSU only temperature profile retrieval step to produce the state $X^{0}$ which is used for initial cloud clearing. Such a step is not performed in Version $5 \mathrm{AO}$.

The cloudy regression can produce biased initial states, especially if it is not followed by an AMSU-A retrieval step. If the state $T^{0}(p)$ used to derive $R_{i, C L R}^{0}$ were biased (say too warm), incorrect values of $\eta_{k}^{0}$ would be determined which would result in $\hat{R}_{i}^{0}$ being too large, which in turn would result in the retrieved $T^{1}(p)$ being too warm. Chahine has shown that it is optimal to use only longwave $(15 \mu \mathrm{m}$ and $12 \mu \mathrm{m})$ channels for cloud clearing, and shortwave $4.2 \mu \mathrm{m}$ channels for the determination of temperature profiles. This is done so as to minimize the bias in retrieved temperature profiles $X^{n+1}$ resulting from biases in the temperature profile $X^{n}$ used to determine $\eta_{k}$. Up to Version 4 , most $4.2 \mu \mathrm{m}$ channels could not be utilized during the day because these channels are affected by non-Local Thermodynamic Equilibrium (non-LTE) which was not accounted for in the Version 4 Radiative Transfer Algorithm (RTA) ${ }^{4}$. Therefore, the optimal combination of channels for cloud clearing and sounding purposes was not used in Version 4, in which most temperature sounding channels were in the $15 \mu \mathrm{m} \mathrm{CO}_{2}$ band.

A significant improvement over the AIRS Science Team Version 4 retrieval algorithm is the use of a new RTA in Version 5 which contains improved characterization of atmospheric absorption characteristics, and more significantly, accounts for effects of non-LTE ${ }^{8}$. This for the first time enabled the use of almost all shortwave $\mathrm{CO}_{2}$ channels in the temperature profile retrieval step of the physical retrieval algorithm, both day and night. This new RTA also contains a better parameterization of the absorption characteristics of the AIRS channels as a function of atmospheric temperature and constituent profile as a function of satellite zenith angle. Stratospheric $15 \mu \mathrm{m} \mathrm{CO}$ channels are included in the temperature profile retrieval step in Version 5 because radiances in these channels are unaffected by clouds in the field of regard. An analogous set of temperature sounding channels is also used in Version 5.24.

\section{CHANNELS AND FUNCTION USED IN DIFFERENT STEPS OF VERSION 5.24}

Figure 2 shows a typical AIRS brightness temperature spectrum and includes the channels used in each Version 5.24 and Version 5.24 AO for cloud clearing, and in each of the different steps of the AIRS physical retrieval algorithm. The channels used in each step are summarized below.

\subsection{Temperature profile retrievals}

The location and number of channels used in the temperature profile retrieval step (red stars) are significantly different from those of Version $4^{3}$. The major difference is in the incorporation of non- $\mathrm{LTE} \mathrm{CO}_{2}$ temperature sounding channels in the spectral region $2358 \mathrm{~cm}^{-1}-2386 \mathrm{~cm}^{-1}$ in the temperature profile retrieval step, which now uses 49 channels between $2197 \mathrm{~cm}^{-1}$ and $2395 \mathrm{~cm}^{-1}$ that are sensitive to both stratospheric and tropospheric temperatures, as well as 53 stratospheric sounding channels between $667 \mathrm{~cm}^{-1}$ and $713 \mathrm{~cm}^{-1}$. Tropospheric sounding longwave $\mathrm{CO}_{2}$ channels are now used only for cloud clearing (yellow stars), and are no longer used in the temperature profile retrieval step. These same channels are also the ones used in the cloud parameter retrieval step ${ }^{3}$. Version 5.24 includes AMSU-A channels 3, 6 and 8-14 in the temperature profile retrieval step while Version 5 AO does not. Versions 4 and 5 included AMSU-A channels 4 and 5 in the temperature profile retrieval step, but they became noisy in 2007 and are no longer used. AMSU-A channel 7 was noisy at launch and was never used in any step of the retrieval process.

\subsection{Surface parameter retrievals as done in Version 4 and Version 5}

In Version 5, as in Version 4, 25 channels are used in the surface parameter retrieval step, 15 of which are between 759 $\mathrm{cm}^{-1}$ and $1228 \mathrm{~cm}^{-1}$, and the remainder are between $2450 \mathrm{~cm}^{-1}$ and $2659 \mathrm{~cm}^{-1}$. These channels are used to determine simultaneously surface skin temperature $T_{s}$, surface spectral emissivity $\varepsilon_{v}$, and short-wave surface bi-directional reflectance $\rho_{v}$. 


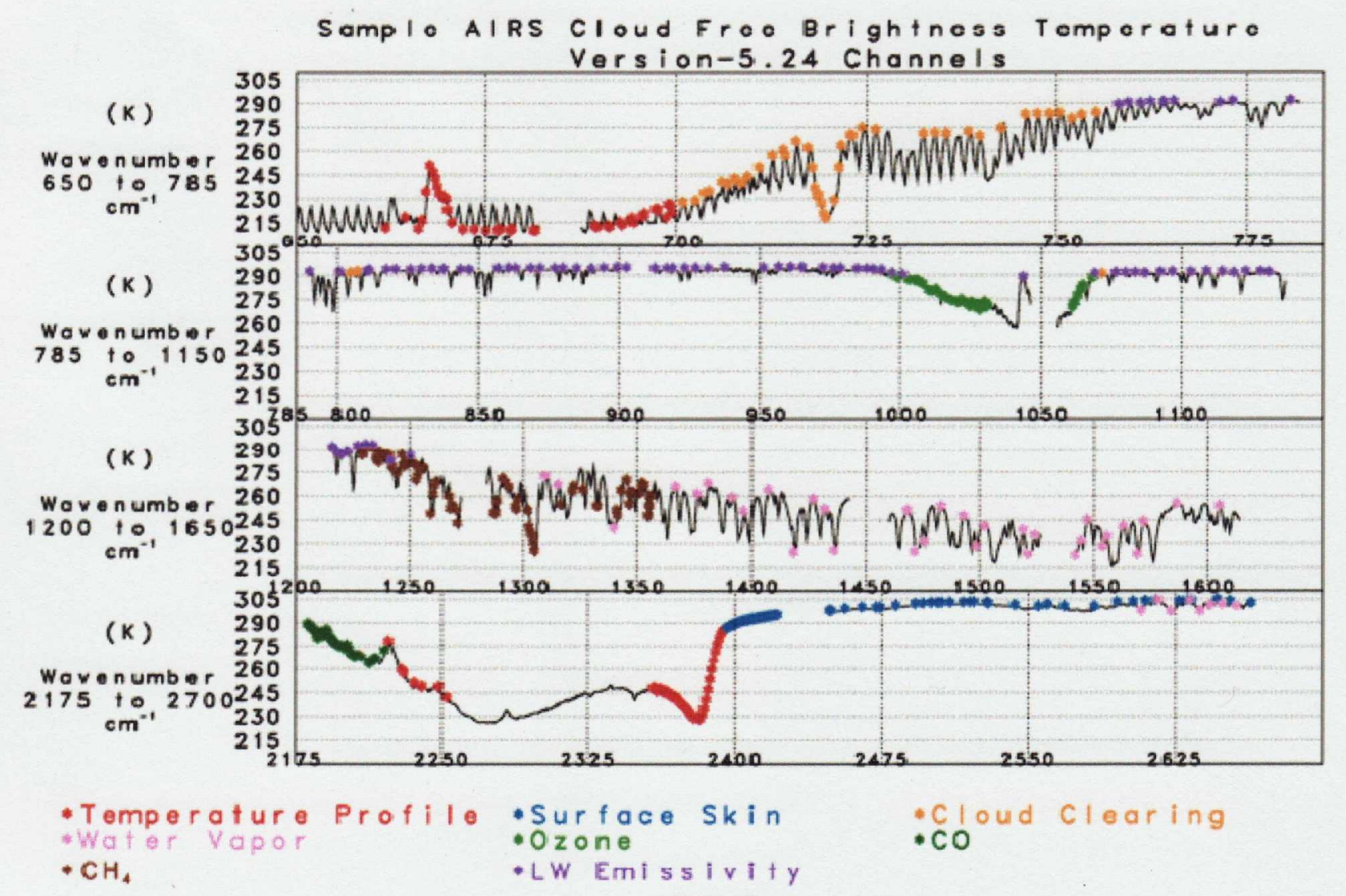

Figure 2

Given an initial surface spectral emissivity guess $\varepsilon_{v}^{0}$, the final surface spectral emissivity $\varepsilon_{v}$ is expressed in Version 5 as

$$
\varepsilon_{v}=\varepsilon_{v}^{0}+\sum_{i=1}^{N_{F}} A_{i} F_{i}(v)
$$

and the final surface bi-directional reflectance $\rho(v)$ is expressed as

$$
\rho_{v}=\rho_{v}^{0}+\sum_{i=1}^{N_{G}} B_{i} G_{i}(v)=\frac{\left(1-\varepsilon_{v}^{0}\right)}{\pi}+\sum_{i=1}^{N_{G}} B_{i} G_{i}(v)
$$

$N_{F}$ and $N_{G}$ in Equations $4 \mathrm{a}$ and $4 \mathrm{~b}$ are the numbers of spectral emissivity and spectral surface bi-directional reflectance functions being solved for in the physical retrieval step. Surface bi-directional reflectance is solved for only during the day. Therefore, including the surface skin temperature which is also solved for in the surface parameter retrieval step, a total of $1+N_{F}+N_{G}$ unknown coefficients are solved for during the day and $1+N_{F}$ unknown coefficients are solved for at night. The emissivity perturbation functions $F$ and $G$ are triangles linear in frequency. In the case of a single function in a spectral region, the correction to the initial guess is constant in frequency. Otherwise, the correction is piecewise linear. 
In Version $4, N_{F}=2$ and there is 1 spectral emissivity perturbation function solved for covering the longwave portion of the spectrum and 1 function covering the shortwave portion of the spectrum, with unknown coefficients $A_{1}\left(A_{\ell w}\right)$ and $A_{2}\left(A_{s w}\right)$. In Version 5, $N_{F}=4$, with coefficients of 3 longwave emissivity perturbation functions being solved for as well as of 1 shortwave emissivity perturbation function. In both Version 4 and Version $5, N_{G}=1$.

Over land, $\varepsilon_{v}^{0}$ is provided by a cloud cleared regression step ${ }^{5}$. Over non-frozen ocean, $\varepsilon_{v}^{0}$ is set equal to the values found in the AIRS Science Team ocean emissivity model. That model is re-interpolated from calculations by Paul VanDelst ${ }^{9}$ using algorithms by Wu referenced in Wu and Smith ${ }^{10}$.

\subsection{Improved surface parameter retrieval methodology used in Version 5.24}

The basic surface parameter retrieval methodology used in Version 5.24 is very similar to that used in Version 5 with two exceptions. The form of the spectral emissivity and surface bi-directional reflectance perturbations has been modified from that shown in equation 4 to a more stable multiplicative form

$$
\left(1-\varepsilon_{v}\right)=\left(1-\varepsilon_{v}^{0}\right)\left(1+\sum A_{i} F_{i}(v)\right)
$$

and

$$
\rho_{\nu}=\rho_{\nu}^{0}\left(1+\sum B_{i} G_{i}(v)\right)
$$

More significantly, 61 channels between $2395 \mathrm{~cm}^{-1}$ and $2664 \mathrm{~cm}^{-1}$ are now used in the surface skin temperature retrieval step, and 3 shortwave emissivity perturbation functions $\left(N_{F}=3\right)$ and 3 shortwave spectral bi-directional reflectance perturbation functions $\left(N_{G}=3\right)$ are now solved for simultaneously with $T_{s}$. These channels are shown in dark blue in Figure 2. 79 channels, between $758 \mathrm{~cm}^{-1}$ and $1250 \mathrm{~cm}^{-1}$, are now used in the subsequent surface spectral emissivity retrieval step, in which 3 longwave emissivity perturbation functions are solved for $\left(N_{F}=3\right.$ as in Version 5). In this step, $T_{s}$ is held fixed at the value determined from the previous skin temperature retrieval step. These channels are indicated by purple stars in Figure 2.

\subsection{Constituent profile retrievals}

As with earlier versions, constituent profile retrievals are performed in separate steps, each having their own set of channels and functions ${ }^{2,3}$. Figure 2 shows in different colors the Version 5.24 channels used in each of these retrieval steps. The $q(p)$ retrieval (pink stars) uses 33 channels in the spectral ranges $1377 \mathrm{~cm}^{-1}$ to $1605 \mathrm{~cm}^{-1}$ and $2608 \mathrm{~cm}^{-1}$ to $2656 \mathrm{~cm}^{-1}$; the $O_{3}(p)$ retrieval (green stars) uses 41 channels between $997 \mathrm{~cm}^{-1}$ and $1069 \mathrm{~cm}^{-1}$; the $C O(p)$ retrieval (green stars) uses 36 channels between $2181 \mathrm{~cm}^{-1}$ and $2221 \mathrm{~cm}^{-1}$; and the $\mathrm{CH}_{4}(p)$ retrieval (brown stars) uses 71 channels between $1230 \mathrm{~cm}^{-1}$ and $1356 \mathrm{~cm}^{-1}$.

\section{VERSION 4 AND VERSION 5 QUALITY CONTROL}

The AIRS Science Team Version 4 retrieval algorithm introduced for the first time the concept of different quality control for different geophysical parameters derived from a given sounding ${ }^{4}$. With regard to temperature profile, there were 3 different flags: 1) Stratosphere Good, i.e, $T(p)$ is good down to $200 \mathrm{mb} ; 2$ ) Mid-Troposphere Good, i.e., $T(p)$ is good down to $\mathrm{p}_{\text {surf }} / 2 ; 3$ ) and Lower Troposphere Good, i.e., $T(p)$ is good down to $\mathrm{p}_{\text {surf. Version }} 5$ further refined the concept so as to determine a pressure, $p_{\text {bess }}$, down to which each retrieval is considered acceptable for data assimilation purposes. The pressure $p_{\text {best }}$ is determined based on thresholds of the case-by-case error estimates of $T(p), \delta T(p)$. Version 5 uses an analogous methodology to perform Quality Control on retrieved sea surface temperatures $T_{s}$, based on thresholds of $\delta T_{s}$. Susskind et al. ${ }^{5}$ provides more details about how error estimates are derived and used for Quality Control. 


\section{VALIDATION OF QUALITY CONTROLLED RETRIEVALS}

\subsection{Temperature profiles}

Figure 3 shows statistics for nighttime $1 \mathrm{~km}$ layer mean temperatures for global Quality Controlled Version 4 and Version 5.24 retrievals for January 25, 2003. Figure 3a shows the percent of all nighttime cases accepted in a given layer. Percent yields using the Version 5 Quality Control thresholds on Version 5 retrievals are shown in Figure 3a in red (dark) and those using the Version 4 thresholds on Version 4 retrievals are shown in gray (light). The percent accepted at $70 \mathrm{mb}$ in Version 4 represents the percent of all cases that passed the Stratosphere Good Test. Version 5.24 accepts all cases above $30 \mathrm{mb}$, and applies Quality Control based on thresholds of error estimates starting at $30 \mathrm{mb}$. Results are shown including all cases down to $p_{\text {bess }}$. Quality Controlled Version 5.24 retrievals have a much higher yield beneath $200 \mathrm{mb}$ than Version 4. Increasing spatial coverage of retrievals is very important for both data assimilation purposes and climate and process studies, provided their accuracy is sufficient for the appropriate application. Quality Controlled Version 5.24 retrievals have a slightly lower yield between $100 \mathrm{mb}$ and $200 \mathrm{mb}$ than Version 4. This is because not all cases passing the Version 4 Stratospheric Temperature Test were actually good all the way down to $200 \mathrm{mb}$. Version 5.24 also has a higher yield above $100 \mathrm{mb}$ because many cases in which the Version 4 retrieval was found not acceptable in the lower stratosphere were actually of high quality in the mid-upper stratosphere.

Figure $3 \mathrm{~b}$ shows the RMS of $1 \mathrm{~km}$ layer mean temperature differences between Quality Controlled Version 5.24 retrievals (red and pink), or Version 4 retrievals (black and gray), and the collocated ECMWF analysis. Dark colors indicate statistics for appropriate retrieval layers selected using the Version 5 (dark) Quality Control, and the light colors indicate statistics for those layers selected using the Version 4 (light) Quality Control. The most meaningful comparisons between Version 5.24 and Version 4 retrievals are made using a common ensemble of cases, i.e., pink vs. gray or red vs. black. Version 5.24 retrievals are considerably more accurate than the corresponding set of Version 4 retrievals in the harder (dark) cases at all levels beneath $70 \mathrm{mb}$ with a minor exception near $600 \mathrm{mb}$. This improvement is smaller in the lower yield (presumably easier cloud condition) cases accepted by Version 4 standards.

Figures $4 \mathrm{a}$ and $4 \mathrm{~b}$ show analogous results for daytime cases. There is no appreciable difference in the results shown during the day compared to the night. This shows that the effects of non-LTE on the radiances is well accounted for by the new RTA. In addition, it shows that effects of solar radiation reflected by clouds is accounted for by the cloud clearing methodology which produces $\hat{R}_{i}$. Finally, the effects of solar radiation reflected by the surface is also well accounted for by the retrieval of $\rho_{i}$, and it's use in the computation of expected radiances $R_{i, C L R}$.

The improvement in Version 5 retrieval accuracy compared to Version 4 is a result of at least two factors, both related to the use of $4.2 \mu \mathrm{m} \mathrm{CO}$ tropospheric sounding channels in the Version 5 temperature profile retrieval step compared to the combined use of $4.2 \mu \mathrm{m}$ and $15 \mu \mathrm{m} \mathrm{CO}_{2}$ channels in the Version 4 temperature profile retrieval step. The main reason for this choice of channels in Version 5 is that cloud clearing errors will result in smaller errors in cloud cleared brightness temperatures $\Delta \widehat{\Theta}_{i}$ for $4.2 \mu \mathrm{m}$ channels than for the $15 \mu \mathrm{m}$ channels. This principle allows for the determination of potentially more accurate temperature soundings under more difficult cloud conditions. This is demonstrated in Figures $3 \mathrm{~b}$ and $4 \mathrm{~b}$ because the relative improvement in Version 5 retrieval accuracy over Version 4 retrieval accuracy is considerably larger in the more difficult cloud cases (dark lines) than in the easier cloud cases (light lines). This was not the only reason for improvement of Version 5 temperature profiles compared to Version 4 however. The inherent temperature sounding capability of the $4.2 \mu \mathrm{m}$ channels may actually be better than those of the $15 \mu \mathrm{m}$ channels, as indicated by the fact that Version 5 results are better in the mid-upper troposphere for easier cases as well.

Version 5 Quality Controlled temperature profile sounding accuracy is also considerably better than that of Version 4 between $70 \mathrm{mb}$ and $200 \mathrm{mb}$. Part of this is due to the fact, alluded to previously, that not all "Stratosphere Good cases" are good down to $200 \mathrm{mb}$, as assumed in Version 4. These cases of poorer upper tropospheric soundings most likely contain very high clouds which are not appropriately screened in the Version 4 Quality Control methodology. Version 5 retrievals between $70 \mathrm{mb}$ and $200 \mathrm{mb}$ using Version 4 Quality Control also perform better than those produced in Version 4. This appears to be partly due to better Version 5 performance under more difficult cloud conditions. In addition, it may also indicate better upper tropospheric sounding capability contained in the $4.2 \mu \mathrm{m}$ sounding channels as compared to the $15 \mu \mathrm{m}$ sounding channels. 


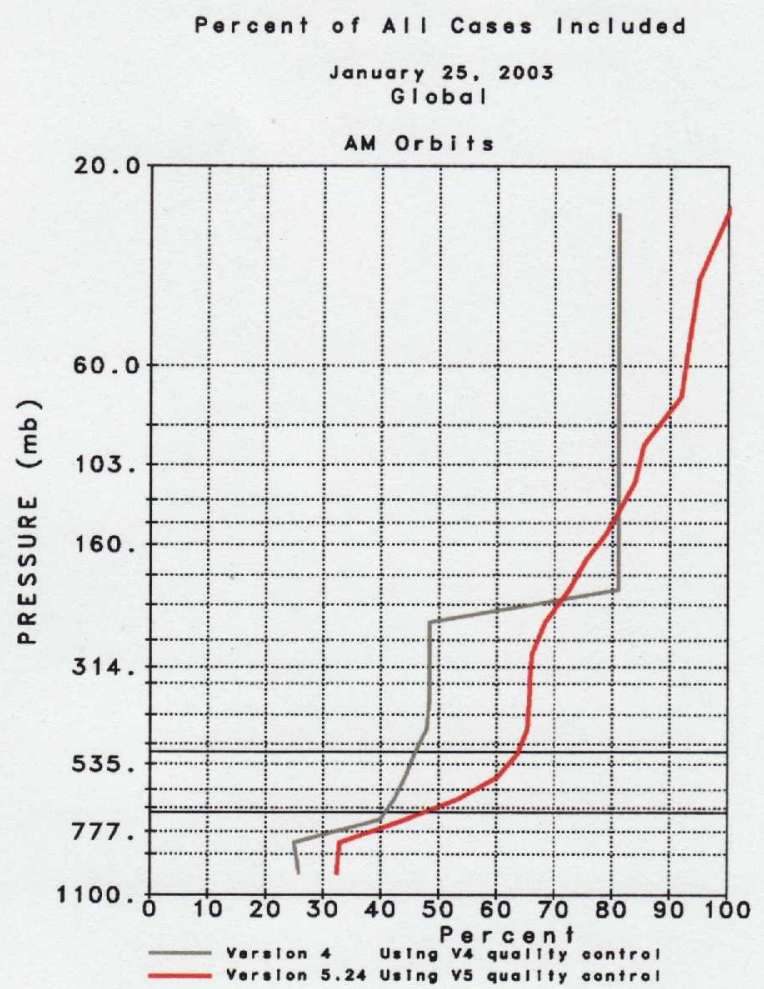

Figure $3 \mathrm{a}$

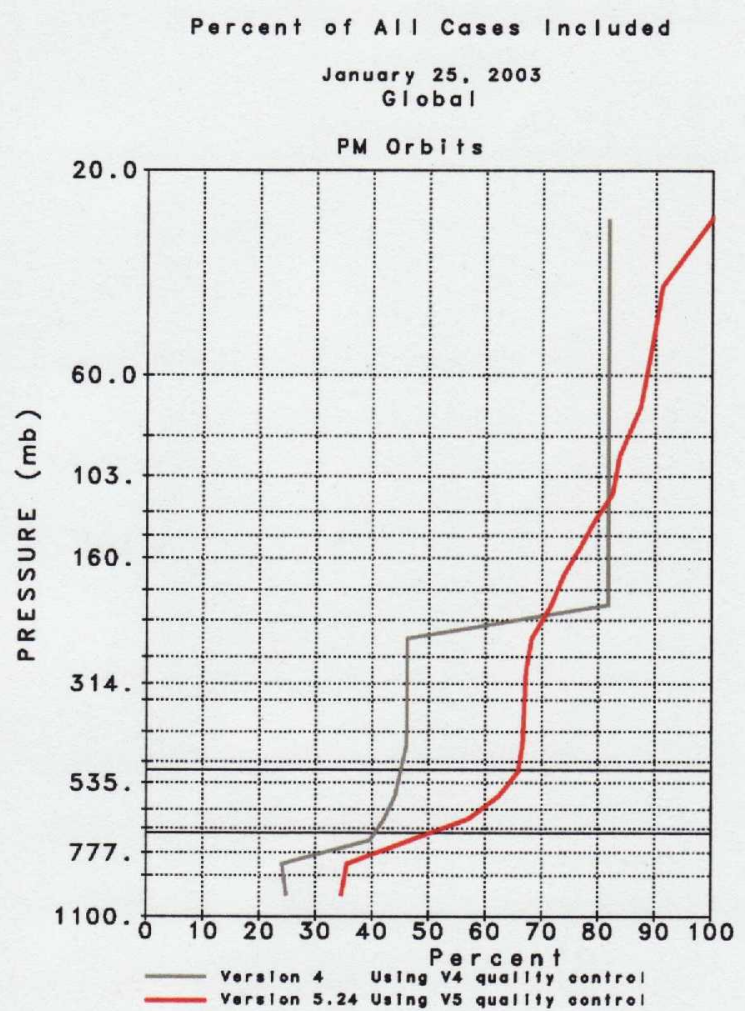

Figure $4 a$

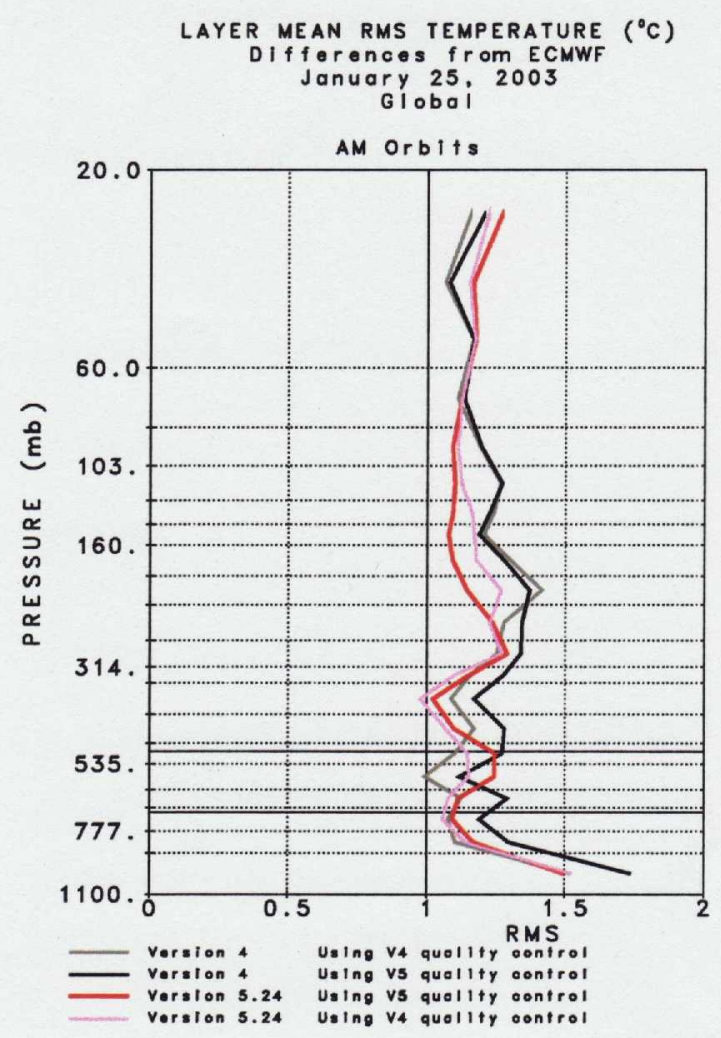

Figure $3 b$

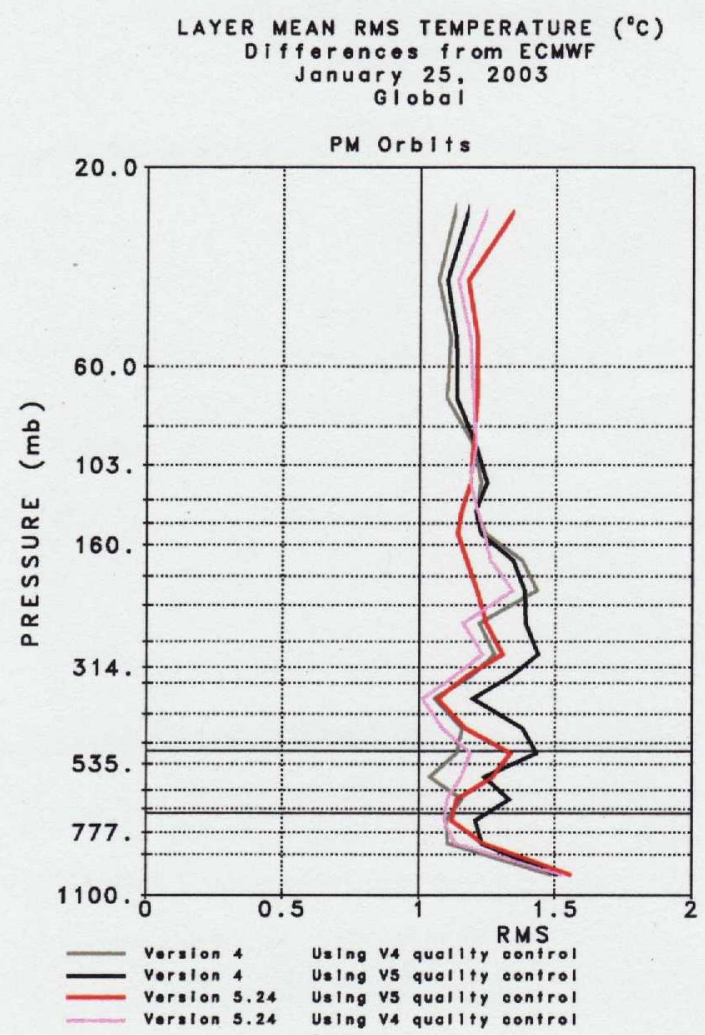

Figure $4 b$ 


\subsection{Quality Controlled ocean surface skin temperature}

The comparisons of the accuracy of Quality Controlled atmospheric temperature profiles shown in the previous section was made using results of Version 4 on the one hand and Version 5.24 on the other. A major difference between these two sets of results is due to the fact that most of the tropospheric temperature profile information in Version 4 came from the use of $\hat{R}_{i}$ for $15 \mu \mathrm{m} \mathrm{CO}$ channels in the retrieval process, while Version 5.24 did not use $\hat{R}_{i}$ for any tropospheric sounding $15 \mu \mathrm{m} \mathrm{CO}_{2}$ channels. There were also other differences between Version 4 and Version 5.24, as well, such as the Quality Control methodology. The improvement in the ability to obtain accurate SST's under more difficult cloud conditions by using $\hat{R}_{i}$ for only shortwave window regions to solve for $\mathrm{T}_{\mathrm{s}}$ as compared to combined use of $\hat{R}_{i}$ in both the longwave and shortwave window regions, is best isolated by a comparison of results of Version 5.24 and 5.0, which are otherwise identical to first order.

Figure 5 shows histograms of the counts of errors of quality controlled ocean SST retrievals as a function of SST differences from ECMWF "truth" on January 25, 2003, obtained using Version 5, and Version 5.24. The mean difference from ECMWF, the spatial standard deviation of the difference, the percent of all cases accepted, and the percent outliers (errors more than $3 \mathrm{~K}$ from the mean) are indicated in Figure 5. Results for day and night are shown separately. Version 5.24 Quality Controlled SST retrievals have considerably higher yields with similar standard deviation and percent outliers than the comparable Quality Controlled Version 5 SST's. The improvement is actually greater during the day than at night.
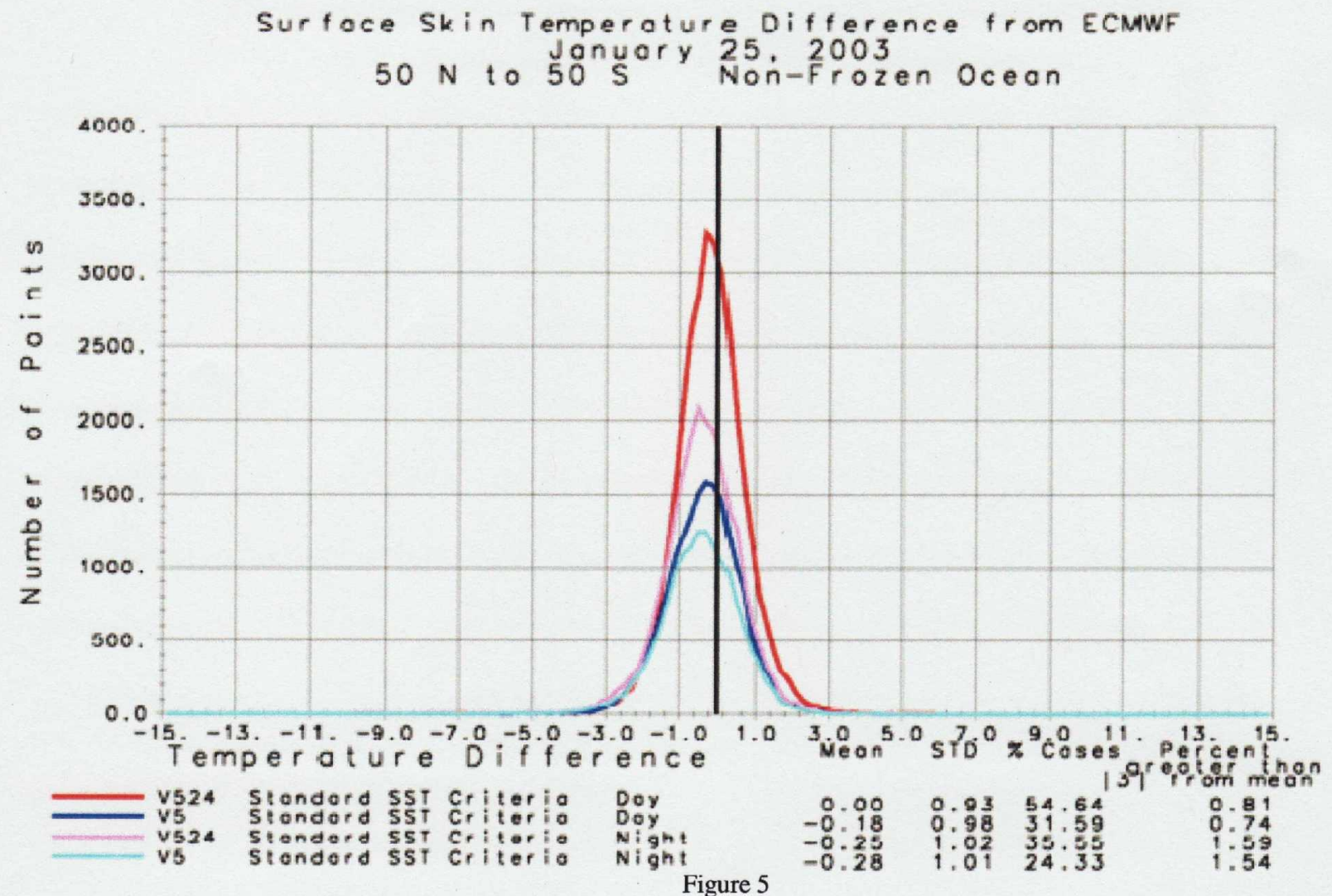

Figure 6 shows the percent of all SST cases $50^{\circ} \mathrm{N}-50^{\circ} \mathrm{S}$ accepted as a function of retrieved fractional cloud cover for both Version 5 and Version 5.24. Results are shown separately for day and night cases. Version 5 Quality Control methodology automatically rejected all retrievals with fractional cloud cover greater than $90 \%$, both with regard to temperature profile and surface skin temperature. Version 5.24 does not have this restriction. All cases shown in this figure were included in the statistics shown in Figure 5. As shown in Figure 5, Version 5.24 has a considerably higher 
percent yield than Version 5, with comparable overall accuracy, both under daytime and nighttime conditions. Version 5.24 Quality Controlled SST retrievals have higher yields than Version 5 at all cloud fractions both day and night. The relative improvement in the ability to determine accurate Quality Controlled SST's between Version 5.24 and Version 5 increases dramatically with increasing cloud cover. For example, at 50\% fractional cloud cover, Version 5.24 accepts roughly $34 \%$ of the cases during the day and $16 \%$ at night, while Version 5 accepts roughly $8 \%$ of the cases under both day and night conditions. This increased yield under more difficult cloud conditions is significant for climate applications.
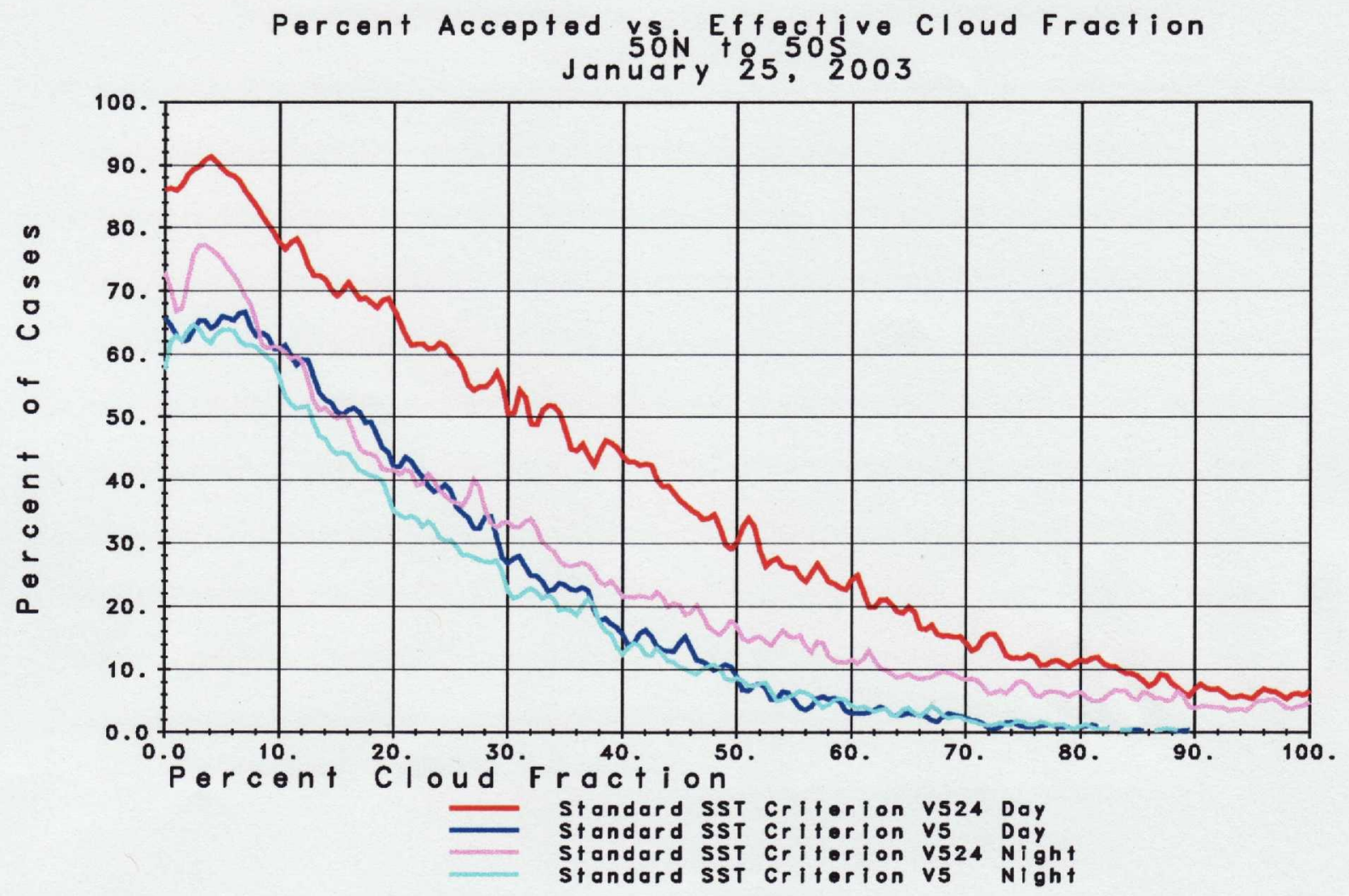

Figure 6

\section{SUMMARY}

This paper shows that improved AIRS Quality Controlled soundings of atmospheric temperature profile $T(p)$ and ocean surface skin temperature $T_{s}$ are obtained by use of cloud cleared radiances $\hat{R}_{i}$ of only shortwave window channels in the determination of $T_{s}$, and primarily of $4.2 \mu \mathrm{m} \mathrm{CO}_{2}$ channels in the determination of $T(p)$. Observed radiances $R_{i}$ are used in the longwave $15 \mu \mathrm{m} \mathrm{CO}$ band and $11 \mu \mathrm{m}$ window in the determination of $\hat{R}_{i}$ for all channels. This improvement in sounding capability exists for relatively clear situations, and is magnified with increasing cloud cover. Results obtained during the day are at least as good, if not better, than those obtained at night. Similar results are obtained in the Version 5.24 AIRS Only system (not shown in this paper).

This improved sounding methodology is made possible as a result of a few factors, both theoretical and instrumental. First of all, use of improved radiative transfer physics allows for the accurate computation of the effects of non-LTE on the observed radiances in the $4.2 \mu \mathrm{m} \mathrm{CO}_{2}$ band during the day. Secondly, the cloud clearing methodology used to analyze AIRS data removes the effects of solar radiation reflected by clouds from the clear column radiances $\hat{R}_{i}$ used to generate the solution. Effects of solar radiation reflected by the surface on the shortwave radiances are accounted for directly as part of the surface parameter retrieval process. Also, improved error estimates and Quality Control methodology allows for the screening of cases in which aspects of the retrieval methodology may have performed poorly. 
Finally, and most significantly, use of these theoretical improvements is made practical because the noise in the AIRS shortwave channels is extremely low. This approach is not practical using IASI data because the IASI noise is too high in the shortwave portion of the spectrum. The findings of this paper are significant with regard to design considerations of future high spectral resolution IR sounders, especially for geostationary (GEO) orbit. It is optimal on such sounders to have spectral coverage beyond $2400 \mathrm{~cm}^{-1}$ with very low noise, to produce accurate soundings under most cloud conditions. It is not essential for advanced IR GEO sounder to be accompanied by a GEO microwave sounder to achieve these results.

\section{REFERENCES}

1. Pagano, T. S., Aumann, H. H., Hagan, D. E. and Overoye, K., "Prelaunch and in-flight radiometric calibration of the Atmospheric Infrared Sounder (AIRS),” IEEE Trans. Geosci. Remote Sensing, 41, 265-273, 2003.

2. Blumstein, D., Chalon, G., Carlier, T., Buil, C., Hebert, P., Maciaszek, T., Ponce, G., Phulpin, T., Tournier, B., Simeoni, D., Astruc, P., Clauss, A., Kayal, G. and Jegou, R., "IASI instrument: technical overview and measured performances," Proc. SPIE 5543, 196, 2004.

3. Susskind, J., Barnet, C. D., and Blaisdell, J. M., "Retrieval of atmospheric and surface parameters from AIRS/AMSU/HSB data in the presence of clouds," IEEE Trans. Geosci. Remote Sensing, 41, 390-409, 2003.

4. Susskind, J., Barnet, C., Blaisdell, J., Iredell, L., Keita, F., Kouvaris, L., Molnar, G. and Chahine, M., "Accuracy of geophysical parameters derived from Atmospheric Infrared Sounder/Advanced Microwave Sounding Unit as a function of fractional cloud cover,” J. Geophys. Res., 111, D09S17, doi:10.1029/2005JD006272, 2006.

5. Susskind, J, Blaisdell, J. M., Iredell, L. and Keita, F., "Improved Temperature Sounding and Quality Control Methodology Using AIRS/AMSU Data: The AIRS Science Team Version 5 Retrieval Algorithm.

6. Chahine, M. T., "Remote sensing of cloudy atmospheres. II. Multiple cloud formations," J. Atmos. Sci., 34, 744-757, 1977.

7. Chahine, M. T., "Remote sensing of cloudy atmospheres. I. The single cloud layer," J. Atmos. Sci., 31, 233-243, 1974.

8. DeSouza-Machado, S. G., Strow, L. L., Hannon, S. E., Motteler, H. E., Lopez-Puertas, M., Funke, B. and Edwards, D. P., "Fast forward radiative transfer modeling of $4.3 \mu \mathrm{m}$ non local therodynamic equilibrium effects for infrared temperature sounders," Geophy. Res. Letters, 34, L01802, doi:10.1029/2006GL026684, 2007.

9. Delst, P. van and Wu, X., "A high resolution infrared sea surface emissivity database for satellite applications, Eleventh International TOVS Study Conf.," Budapest, Hungary, 2000.

10. Wu, X. and Smith, W. L., Emissivity of rough sea surface for 8-13 $\mu \mathrm{m}$ : Modeling and verification," Appl. Opt., 36, 2609-2619, 1997 